\title{
Activation of mTOR signaling leads to orthopedic surgery-induced cognitive decline in mice through $\beta$-amyloid accumulation and tau phosphorylation
}

\author{
WENZHEN SHEN ${ }^{1}$, KELIANG LU ${ }^{2}$, JIAWAN WANG ${ }^{1}$, ANSHI WU ${ }^{1 *}$ and YUN YUE ${ }^{1 *}$ \\ ${ }^{1}$ Department of Anesthesiology, Beijing Chao-Yang Hospital, Capital Medical University, Beijing 100020; \\ ${ }^{2}$ Department of Anesthesiology, Weifang Medical University, Weifang, Shandong 261031, P.R. China
}

Received August 4, 2015; Accepted July 13, 2016

DOI: $10.3892 / \mathrm{mmr} .2016 .5700$

\begin{abstract}
Postoperative cognitive dysfunction (POCD) is a serious complication following surgery, however, the mechanism of POCD remains to be elucidated. Previous evidence has revealed that POCD may be associated with the pathogenesis of neurodegenerative processes. The mammalian target of rapamycin (mTOR) signaling pathway has been reported to be crucial in the pathophysiology of neurodegenerative diseases. However, the implications of mTOR in POCD remains to be fully elucidated. In the present study, western blotting and enzyme-linked immunosorbent assay were used to determine the expression of mTOR and any associated downstream targets; contextual fear conditioning was used to estimate the learning and memory ability of mice. Using an animal model of orthopedic surgery, it was found that surgical injury impaired hippocampal-dependent memory and enhanced the levels of phosphorylated mTOR at Serine-2448, phosphorylated 70-kDa ribosomal protein S6 kinase (p70S6K) at Threonine-389 with accumulation of $\beta$-amyloid $(\mathrm{A} \beta)$ and hyperphosphorylated tau at Serine-396, compared with the control group. Pretreatment with rapamycin, an mTOR inhibitor, restored the abnormal mTOR/p70S6K signaling induced by surgery, attenuated the accumulation of $A \beta$ and reduced the phosphorylation of tau protein. Rapamycin also reversed the surgery-induced cognitive dysfunction. The results of the present study suggested that the surgical stimulus activated mTOR/p70S6K signaling excessively, and that the inhibition of mTOR signaling with rapamycin may prevent postoperative
\end{abstract}

Correspondence to: Dr Yun Yue or Dr Anshi Wu, Department of Anesthesiology, Beijing Chao-Yang Hospital, Capital Medical University, 8 Gongtinan Road, Chaoyang, Beijing 100020, P.R. China E-mail: yueyun@hotmail.com

E-mail:wuanshi8@sina.com

*Contributed equally

Key words: cognitive decline, mammalian target of rapamycin, orthopedic surgery, $\beta$-amyloid, tau phosphorylation cognitive deficits, partly through attenuating the accumulation of $A \beta$ and hyperphosphorylation of tau protein.

\section{Introduction}

Postoperative cognitive dysfunction (POCD) is a common complication involving transient or permanent impairment of cognition in elderly patients following surgery (1). POCD affects a wide variety of cognitive domains, including attention, memory, executive function and speed of information processing, resulting in a reduction in the patient's quality of life and contributing substantially to healthcare costs. The International Multicenter Study on POCD reported that, following noncardiac surgery, cognitive impairments occur in $25.8 \%$ of patients 1 week postoperatively and in $9.9 \%$ of patients 3 months postoperatively in patients $>60$ years of age (1). Following cardiac surgery, the short-term (2 weeks postoperatively) rate ranges between 26 and $80 \%$, whereas the long-term rate (5 years following cardiac surgery) is up to $37 \%$ (2). With a steady increase in the geriatric surgical population, postoperative cognitive decline is rapidly becoming a major global health burden (3). Certain risk factors have been identified to contribute to postoperative cognitive dysfunction, including increasing age, anesthetics, surgical intervention and postoperative pain (4). Increasing age and the extent of surgical trauma are the only univocal risk factors $(1,5)$, however, the molecular mechanisms underlying POCD remain to be fully elucidated. There is evidence to suggest that POCD and neurodegenerative diseases, including Alzheimer's disease (AD), may share certain neuropathological and biochemical mechanisms.

Mammalian target of rapamycin (mTOR) is a conserved serine/threonine protein kinase and is a member of the phosphoinositide-3-kinase-related family. It is involved in cell growth, proliferation, metabolism and protein synthesis, which integrates a variety of signals under physiological conditions (6). In the nervous system, mTOR is critical in maintaining brain function. A series of studies have found that mTOR promotes learning and memory formation via the protein synthesis-dependent strengthening of synapses (7). For example, mice deficient in mTOR have impaired learning, memory and social behavior (8), and the dysregulation of 
mTOR has been shown to cause learning deficits (9). Several studies have demonstrated that mTOR signaling may be linked to several neurodegenerative diseases $(10,11)$. Neurodegenerative diseases, including $\mathrm{AD}$, are characterized by the aberrant accumulation of misfolded proteins, leading to memory and cognitive impairment. mTOR signaling has been shown to be involved in the synthesis of $\beta$-amyloid and tau protein. The upregulation of mTOR and p70S6 K has been found to be associated with the accumulation of hyperphosphorylated tau in AD (12), and the inhibition of mTOR by rapamycin has been shown to improve learning and memory abilities, and reduce levels of $\beta$-amyloid by inducing the autophagic removal of proteins $(13,14)$. POCD is a prolonged change in cognition, with similar clinical manifestations and physiopathologic mechanisms to other neurodegenerative disorders. Therefore, the present study hypothesized that mTOR signaling may also be involved in the development of POCD.

In the present study, an animal model was used to examine whether surgical trauma leads to the activation of mTOR signaling within the hippocampal area, and whether inhibition of the mTOR signaling pathway within the hippocampal area ameliorates the cognitive impairment. The present study also aimed to determine whether the activation of mTOR signaling causes the accumulation of $A \beta_{1-42}$ and hyperphosphorylated tau protein, which can lead to memory and cognitive impairment.

\section{Materials and methods}

Animals. Male C57BL/6J mice ( $\mathrm{n}=104)$, aged 12-14 weeks, weighing 20-25 g (Vital River Laboratory Animal Technology Co., Ltd., Beijing, China), were used for all experiments. The animals were housed under controlled conditions $\left(21 \pm 2^{\circ} \mathrm{C}\right.$; $50 \pm 10 \%$ humidity, $12: 12 \mathrm{~h}$ light:dark cycle) with access to food and water ad libitum. All mice were allowed to adapt to the environment for 7 days prior to beginning the experiments. All experimental protocols were approved by the animal ethics committee of Capital Medical University (Beijing, China), and were in accordance with the guidelines for animal experiments of the local Animal Care and Use Committee.

Experiment 1 protocol. Based on preliminary time course experiments (15-17), to investigate the effects of orthopedic surgery on the activation of mTOR, $\beta$-amyloid accumulation and tau phosphorylation, the present study examined the changes of relevant proteins. The animals were divided into three groups ( $n=8 /$ group). Normal, untreated animals were used as a control group. In the surgery group, the mice underwent orthopedic surgery of left hindpaw under isoflurane anesthesia and analgesia. In the sham surgery group, mice received the same anesthesia and analgesia as the surgery group. The mice in each group received contextual fear conditioning (CFC) to estimate the learning and memory abilities of the mice, following which the animals were sacrificed by cervical dislocation, and hippocampal tissue samples were obtained for further experiments. Animals were anesthetized prior to cervical dislocation through a single intraperitoneal injection of chloral hydrate $(10 \% ; 0.3 \mathrm{ml} / 100 \mathrm{~g})$.

Experiment 2 protocol. The experiments described above showed the activation of mTOR signaling with $\beta$-amyloid accumulation and tau phosphorylation in response to surgical stimulation. Therefore, subsequent experiments were performed to examine the effects of pretreatment with rapamycin, an inhibitor of mTOR, on postoperative cognitive function, and to determine the levels of $A \beta_{1-42}$ and tau phosphorylation. This was performed to confirm whether the hyperactivation of mTOR signaling was involved in cognitive defects following surgery via the upstream regulation of $A \beta_{1-42}$ and the phosphorylation of tau. To investigate the effects, the mice were divided into four groups ( $n=8 /$ group): Ctrl group (mice received injections of vehicle without surgery), Ctrl+rapa group (mice received rapamycin pretreatment without surgery), Sur group (mice received orthopedic surgery with injections of vehicle) and Sur+rapa group (mice received orthopedic surgery with rapamycin pretreatment). The animals received CFC 1 day following surgery, and the mice were then sacrificed by cervical dislocation for western blot and enzyme-linked immunosorbent assays.

Anesthesia, orthopedic surgery and pharmacological treatment. Anesthesia was prepared using a procedure described by Degos et al (18). In brief, the animals were placed in a sealed plastic box and anesthesia was induced with $5 \%$ isoflurane mixed with air. The anesthesia was maintained with $1.2-1.5 \%$ isoflurane, which was delivered through a nose cone to the mouse for $15 \mathrm{~min}$. The gas concentrations and respiratory rate were continuously monitored using a multi-function monitor (Datex-Ohmeda, Helsinki, Finland). In the surgery group, an open tibial fracture of the left hind paw with intramedullary fixation was performed in aseptic conditions under general anesthesia with isoflurane $(19,20)$. Buprenorphine was used to provide supplemental analgesia $(0.1 \mathrm{mg} / \mathrm{kg}$ administered subcutaneously). The surgical aspect of the left hindpaw was sterilized with povidone-iodine, and a median incision on the surgical region was made. Following incision, a $0.38 \mathrm{~mm}$ pin was inserted in the intramedullary canal, the periosteum was stripped and the wound was irrigated. Finally, the skin of the wound was closed with 5-0 nylon sutures and covered with antibiotic ointment. Following surgery, the mice were moved back to their original cage for recovery with a sufficient supply of food and water. Throughout the entire anesthesia and surgical procedures, all vital signs were monitored, and blood gas analysis was performed following anesthesia and surgery. For inhibition of the mTOR signal, rapamycin was used to reduce the activity of mTORC1. The dose of rapamycin used was selected based on previous studies $(21,22)$. The mice received intraperitoneal injections of $0.5 \mathrm{mg} / \mathrm{kg} /$ day rapamycin for 3 days prior to undergoing orthopedic surgery, with the final dose administered $2 \mathrm{~h}$ prior to surgery. In the negative control group, animals received an intraperitoneal injection of $0.5 \mathrm{mg} / \mathrm{kg} /$ day rapamycin for 3 days without surgery.

$C F C$. The animals were transported to the laboratory at least $2 \mathrm{~h}$ prior to $\mathrm{CFC}$ training. For $\mathrm{CFC}$, a clear plexiglas chamber was placed in a soundproof box and a camera (Meidi Electronic Co., Ltd., Shenzhen, China) was fixed to the top of the box to capture videos of each animal during CFC training using ANY-maze software (Stoelting Co., Wood Dale, IL, USA). Foot shocks were delivered through a grid floor, which 
comprised 28 stainless steel bars. Prior to and following each session, the chambers were cleaned using pine solution.

Training was performed $24 \mathrm{~h}$ prior to surgery. The animals were allowed free movement in the chambers for $5 \mathrm{~min}$ prior to training, following which they received three tone $(2,000 \mathrm{~Hz}$; $90 \mathrm{~dB})$-electric shock $(0.85 \mathrm{~mA}$ for $2 \mathrm{sec})$ pairings, which were separated by $60 \mathrm{sec}$. Following fear establishment, the fear response of the animals was measured based on freezing times. The percentage of freezing time was used to reflect the hippocampal-dependent memory. Following the different treatments, the mice were returned to the training environment to assess the contextual fear memory. During CFC assessment test, each mouse was placed once again into the chamber for three 3 min without tone or shock. Freezing time was measured by two observers blinded to the group assignments.

Tissue preparation. For western blot analysis and the enzyme-linked immunosorbent assay, the mice were sacrificed under deep anesthesia. The hippocampus was removed and stored at $-80^{\circ} \mathrm{C}$.

Western blot analysis. The hippocampal tissues were homogenized in RIPA buffer containing protease and phosphatase inhibitors, and then centrifuged at $4^{\circ} \mathrm{C}$ at $12,000 \mathrm{~g}$ for $25 \mathrm{~min}$. The concentration of protein in the supernatants was determined using a Bradford protein assay kit (Beyotime Institute of Biotechnology Co., Ltd., Shanghai, China). Equal quantities of the protein samples (30 $\mu \mathrm{g}$ per sample) were denatured at $100^{\circ} \mathrm{C}$ for $5 \mathrm{~min}$, and were then separated by $8-12 \%$ sodium dodecyl sulfate-polyacrylamide gel electrophoresis and transferred electrophoretically onto a polyvinylidene fluoride membrane (EMD Millipore, Billerica, MA, USA). The membranes were blocked using 5\% skim milk-Tris-buffered saline (TBS) buffer for $60 \mathrm{~min}$ and then incubated with the following primary antibodies: Rabbit polyclonal anti-mTOR (1:1,000; cat. no. 2972; Cell Signaling Technology, Inc., Boston, MA, USA), rabbit polyclonal anti-phosphorylated (phospho)-mTOR (Ser2448; 1:1,000; cat. no. 2971; Cell Signaling Technology, Inc.), rabbit polyclonal anti-P70S6K (1:1,000; cat. no. 9202; Cell Signaling Technology, Inc.), rabbit polyclonal anti-phospho-P70S6K (Thr389; 1:1,000; cat. no. 9205; Cell Signaling Technology, Inc.), rabbit polyclonal anti-Tau (1:500; cat. no. YT4554; Immunoway, Newark, DE, USA) and rabbit polyclonal anti-phospho-Tau (Ser396; 1:500; cat. no. YP0263; Immunoway) overnight at $4^{\circ} \mathrm{C}$. Following three washes (10 min each) in TBS with Tween solution, the membranes were incubated with horseradish peroxidase conjugated goat anti-rabbit IgG (1:2,000; Beijing Zhongshan Golden Bridge Biotechnology Co., Ltd., Beijing, China) secondary antibodies at room temperature for $1 \mathrm{~h}$. The bands were treated with an enhanced chemiluminescence detection kit (EMD Millipore), and the intensity of each band was quantified by densitometric analysis. The relative expression levels of protein were normalized by GAPDH $(1: 1,000$; cat. no. 5174; Cell Signaling Technology, Inc.).

Enzyme-linked immunosorbent assay. The hippocampal tissue samples were weighed and sonicated in phosphate-buffered saline with $50 \mathrm{mM}$ protease inhibitor cocktail, followed by centrifugation at $20,000 \mathrm{~g} 4^{\circ} \mathrm{C}$ for $10 \mathrm{~min}$. The supernatant

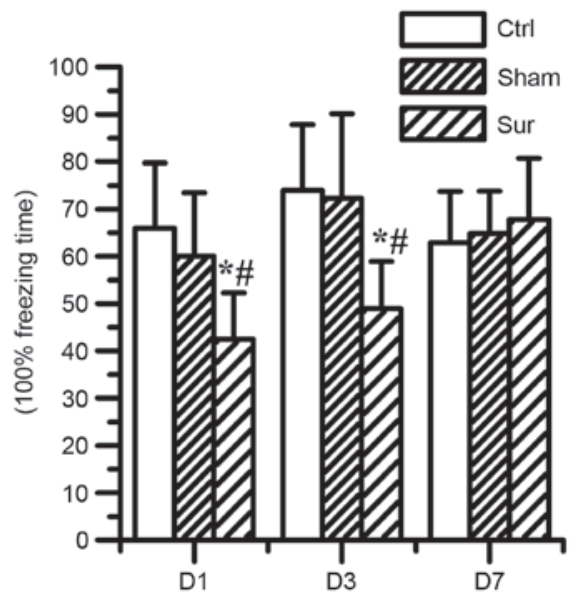

Figure 1. Effects of orthopedic surgery on hippocampal-dependent memory, measured as the percentage of freezing time. Surgery decreased hippocampal-dependent memory on day 1 and day 3 ("P<0.05, compared with $\mathrm{Ctrl}$; ${ }^{\#} \mathrm{P}<0.05$, compared with Sham). In the Sham group, no significant difference was observed in the freezing time following isoflurane anesthesia, compared with the $\mathrm{Ctrl}(\mathrm{P}>0.05)$. Values are presented as the mean \pm standard deviation ( $\mathrm{n}=8$ for each group). Ctrl, control group; Sham, sham surgery group; Sur, surgery group. D1, day 1; D3, day 3; D7, day 7 .

was collected to quantify the protein concentration in the samples using a BCA protein assay kit (cat. no. 23225; Thermo Fisher Scientific, Inc., Waltham, MA, USA). Equal quantities of protein sample $(50 \mu \mathrm{g})$ were used for the measurement of $\mathrm{A} \beta_{1-42}$ (Wuxi Donglin Sci \& Tech Development Co., Ltd., Jiangsu, China) using an enzyme-linked immunosorbent assay, according to the manufacturer's protocol. The intensity of the color was measured at a wavelength of $450 \mathrm{~nm}$ using an iMark microplate reader (Bio-Rad Laboratories, Inc., Hercules, CA, USA).

Statistical analysis. All data were analyzed using SPSS statistical software, version 18.0 (IBM SPSS, Armonk, NY, USA). The data were expressed as the mean \pm standard deviation, and statistical analysis was performed using one-way analysis of variance followed by Newman-Keuls post-hoc test where appropriate. $\mathrm{P}<0.05$ was considered to indicate a statistically significant difference.

\section{Results}

Orthopedic surgery impairs learning and memory function. To elucidate the effect of surgery on cognitive function, CFC assessment was performed to assess learning and memory function. Compared with the control group and sham group, animals in the surgery group presented with significantly

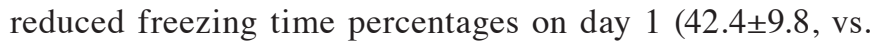
$65.9 \pm 13.8 \%$, respectively; $\mathrm{P}<0.05)$ and day $3(48.95 \pm 9.97$, vs. $73.88 \pm 13.9 \%$, respectively; $\mathrm{P}<0.05)$. No significant difference in freezing time was found between the sham group and control group (Fig. 1).

Orthopedic surgery induces the upregulation of $m T O R / p 70 S 6 K$ signaling in the hippocampus. To determine the effects of surgical trauma on mTOR/p70S6K signaling, the present study detected the levels of mTOR and p70S6K in the homogenates 
A

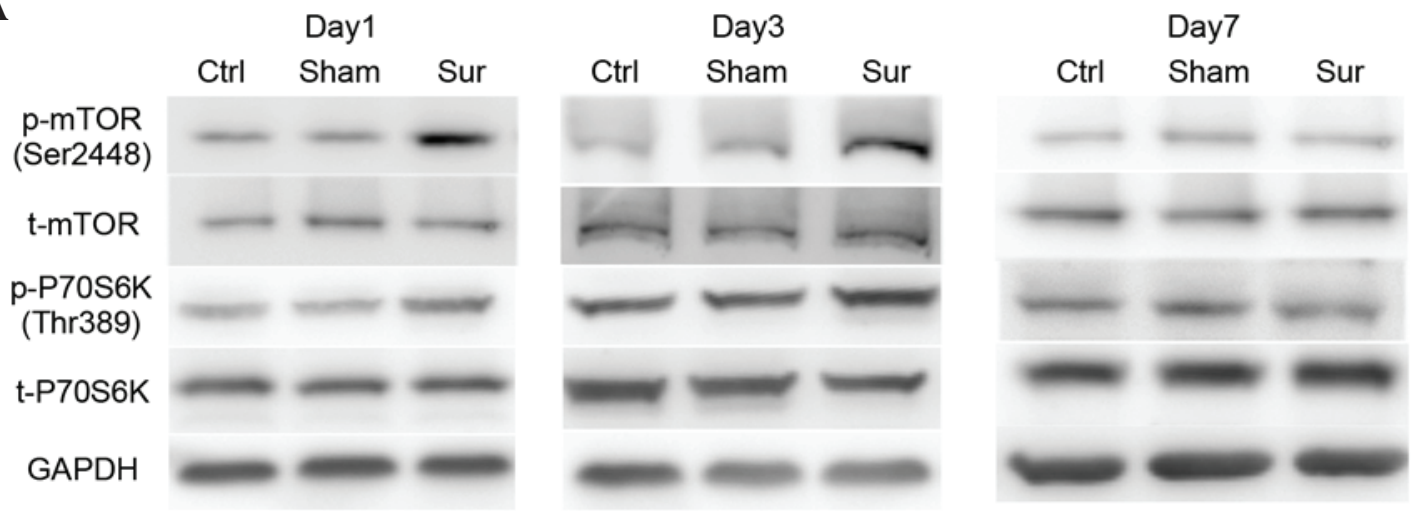

B

C
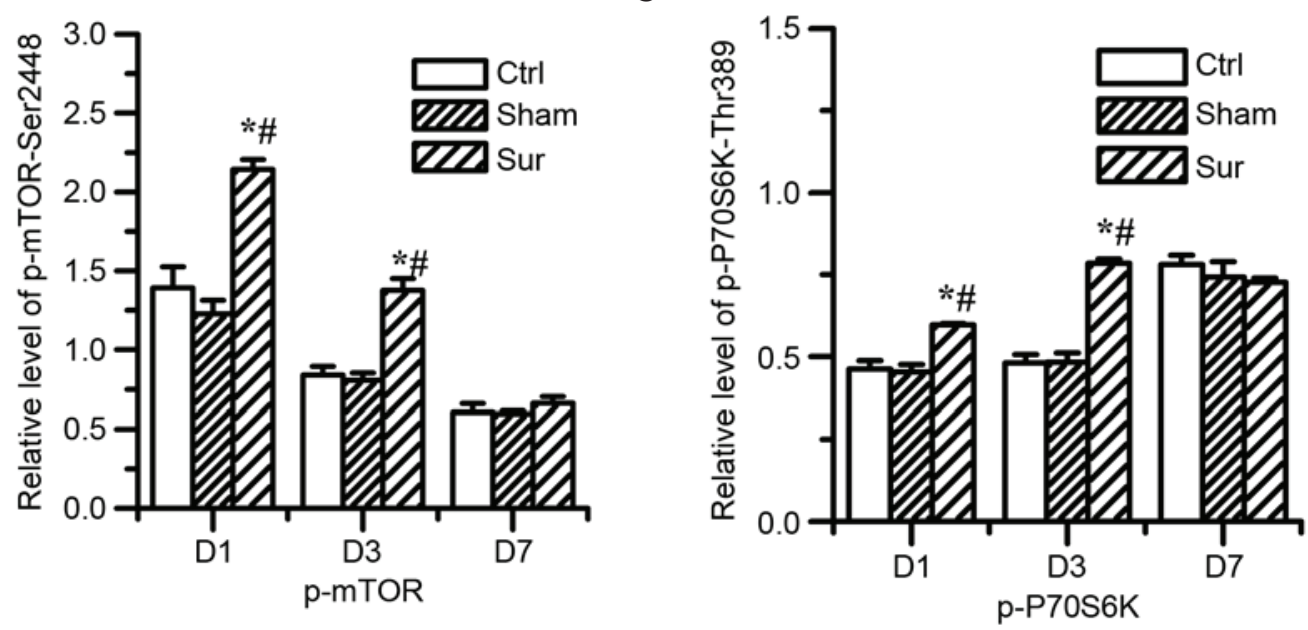

D
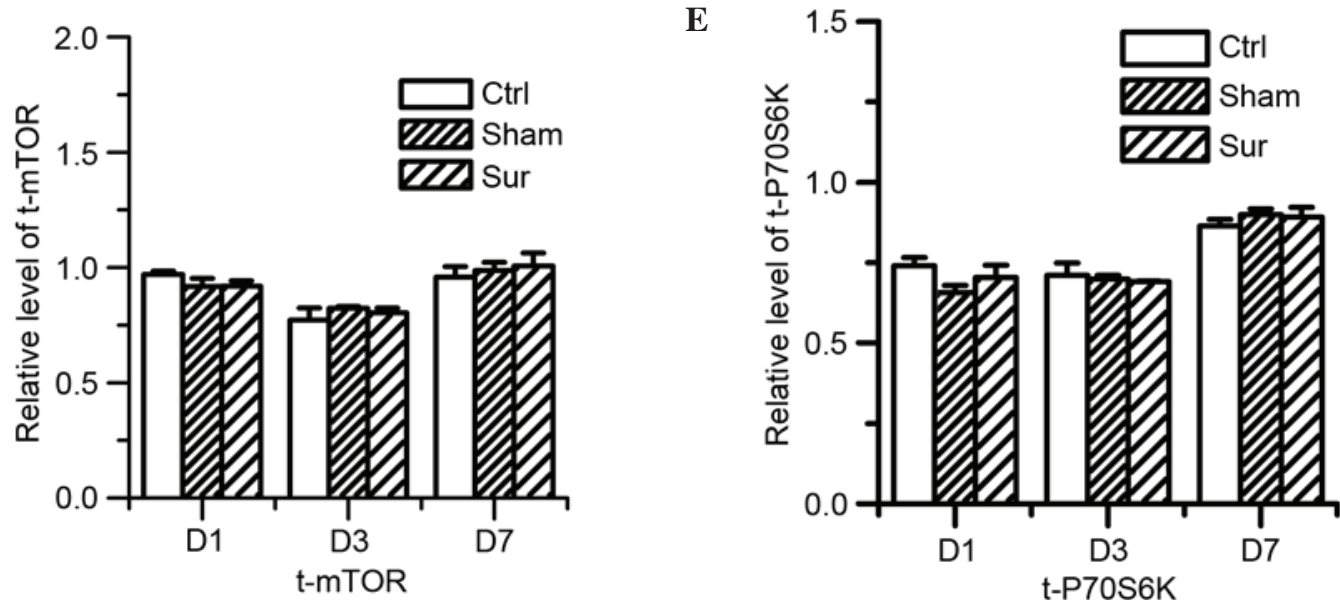

Figure 2. Effects of orthopedic surgery on the activation of mTOR signaling in the hippocampus. The expression levels of mTOR and p70S6K were determined by western blot analysis on days 1, 3 and 7 post-surgery. (A) Typical immunoblot results showed the changes in the levels of p-mTOR (Ser2448), total mTOR, p-p70S6 K (Thr396) and total p70S6K at different points. Quantitative analysis indicated that surgery significantly increased the levels of (B) p-mTOR (Ser2448) and (C) p-p70S6K (Thr396) on days 1 and 3 post-surgery ( ${ }^{*} \mathrm{P}<0.05$, vs. Ctrl; ${ }^{*} \mathrm{P}<0.05$ vs. Sham), however, the protein expression levels of (D) t-mTOR and (E) t-p70S6K, were not affected, and this difference was reversed on day 7 post-surgery. In the Sham group, no significant difference in the levels of p-mTOR (Ser2448) or p-p70S6K (Thr396) were observed following isoflurane anesthesia, compared with the Ctrl (P>0.05, vs. Ctrl). Values are presented as the mean \pm standard deviation ( $\mathrm{n}=8$ for each group). $\mathrm{mTOR}$, mammalian target of rapamycin; $\mathrm{p}-$, phosphoarylated; Ctrl, control group; Sham, sham surgery group; Sur, surgery group; D1, day 1; D3, day 3; D7, day 7.

of the hippocampal tissue using western blot analysis (Fig. 2). Although there were no significant differences in the levels of total mTOR or p70S6K (Fig. 2D and E), the levels of
phospho-mTOR at Ser2448 and phospho-p70S6K at Thr396 were significantly increased on day 1 and day 3 in the hippocampal tissues of the surgery group $(\mathrm{P}<0.05 ; \mathrm{n}=8)$, as shown 


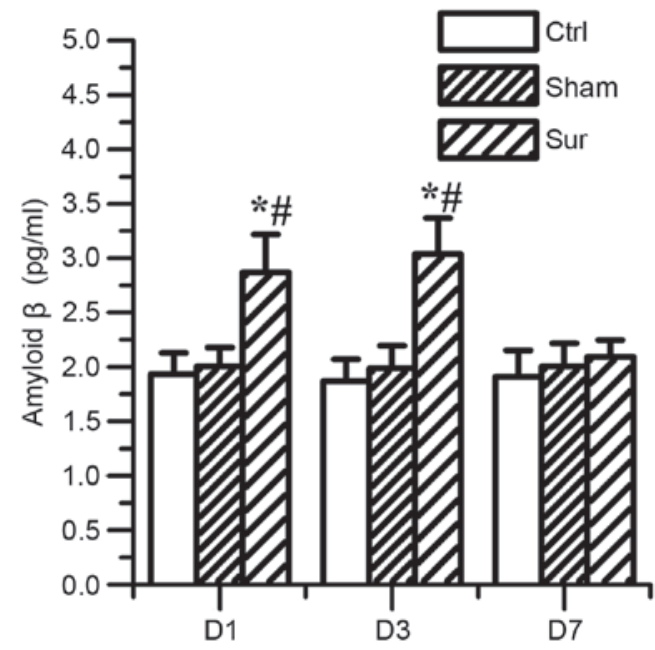

Figure 3. Effects of orthopedic surgery on the levels of $A \beta_{1-42}$ in the hippocampus. The expression of $A \beta_{1-42}$ was determined using an enzyme-linked immunosorbent assay on days 1,3 and 7 post-surgery. The level of $A \beta_{1-42}$ increased significantly on days 1 and 3 post-surgery (" $\mathrm{P}<0.05$, vs. $\mathrm{Ctrl}$ ${ }^{\#} \mathrm{P}<0.05$, vs. Sham). No significant difference was found in the level of $\mathrm{A} \beta_{1-42}$ in the Sham group, compared with the $\mathrm{Ctrl}$ group ( $\mathrm{P}>0.05$, vs. Ctrl). Values are presented as the mean \pm standard deviation ( $n=8$ for each group). $A \beta_{1-42}$, amyloid $\beta$; Ctrl, control group; Sham, sham surgery group; Sur, surgery group; D1, day 1; D3, day 3; D7, day 7.

in Fig. 2B and C. No significant differences were found in the levels of phospho-mTOR at Ser2448 or phospho-p70S6K at Thr396 between the sham surgery group and the control group (Fig. 2B and C). These data indicated that surgery elevated the activation of mTOR signaling on day 1 and day 3. However, the effect of surgery on mTOR signaling was reversed on day 7. Surgical trauma induced the upregulation of mTOR/p70S6K signaling, which suggested that the activation of mTOR signaling may be involved in the development of postoperative dysfunction.

Orthopedic surgery induces $\beta$-amyloid accumulation and tau phosphorylation in the hippocampus. To investigate the effect of orthopedic surgery on the production of $\beta$-amyloid, the present study measured the level of $A \beta_{1-42}$. Surgery increased the production of $\mathrm{A} \beta_{1-42}$ at $24 \mathrm{~h}(2.88 \pm 0.34$, vs. $1.93 \pm 0.19 \mathrm{pg} / \mathrm{ml}$ ) and 3 days post-surgery (3.0 \pm 0.32 , vs. $1.86 \pm 0.20$ pg/ml; $\mathrm{P}<0.05$; n=8; Fig. 3). No significant differences were observed between the sham surgery group and control group $(\mathrm{P}>0.05 ; \mathrm{n}=8)$.

Tau protein hyperphosphorylation is associated with the upregulation of mTOR/p70S6K signaling in the development of AD. The present study also investigated whether tau phosphorylation occurred following surgery accompanying the increased mTOR activity. The results showed that orthopedic surgery upregulated the levels of phosphorylated protein at Ser396 on day 1 and day 3 post-surgery, compared with the sham surgery and control group $(\mathrm{P}<0.05 ; \mathrm{n}=8$; Fig. 4B). However, surgery did not alter the expression of total tau protein (Fig. 4C).

Rapamycin treatment inhibits the abnormal mTOR/p70S6K signaling in the hippocampus induced by orthopedic surgery. As mTOR/p70S6K signaling is hyperactive under the condition of traumatic stimulation and rapamycin is a well known mTOR inhibitor, the present study investigated the effect of rapamycin administration on mTOR/p70S6K signaling. The data showed that rapamycin treatment significantly reduced the levels of phospho-mTOR at Ser2448 and phospho-p70S6K at Thr396 induced by surgical trauma $(\mathrm{P}<0.05$; $\mathrm{n}=8$; Fig. $5 \mathrm{~B}$ and $\mathrm{C})$. However, rapamycin had no effect on the total levels of mTOR or p70S6K (Fig. 5D and E). Rapamycin treatment also reduced the levels of phospho-mTOR at Ser2448 and phospho-p70S6K at Thr396 in the normal control mice $(\mathrm{P}<0.05$; $\mathrm{n}=8$; Fig. $5 \mathrm{~B}$ and C).

Rapamycin treatment attenuates $\beta$-amyloid accumulation and the hyperphosphorylation of tau protein triggered by orthopedic surgery in the hippocampus. In order to investigate whether the hyperactivation of $\mathrm{mTOR} / \mathrm{p} 70 \mathrm{~S} 6 \mathrm{~K}$ signaling was involved in the accumulation of $\beta$-amyloid and hyperphosphorylation of tau protein, the present study examined the effect of rapamycin on the levels of $A \beta_{1-42}$, total tau and phospho-tau. It was found that rapamycin significantly attenuated the production of $\mathrm{A} \beta_{1-42}(2.32 \pm 0.18 \mathrm{pg} / \mathrm{ml})$, compared with the surgery group $(2.99 \pm 0.27 \mathrm{pg} / \mathrm{ml})$, as shown in Fig.6 $(\mathrm{P}<0.01 ; \mathrm{n}=8)$ and attenuated the level of hyperphosphorylated tau at Ser396, $(\mathrm{P}<0.05$; n=8; Fig. 7A and $\mathrm{B})$ which were triggered by surgical injury. However, rapamycin had no effect on the total level of tau protein (Fig. 7A and C). Rapamycin did not affect the levels of $A \beta_{1-42}$, total tau or phospho-tau in the normal control mice (Figs. 6 and 7).

Rapamycin treatment ameliorates learning and memory impairment induced by orthopedic surgery. The present study subsequently investigated whether the increase in mTOR/p70S6K signaling contributed to the cognitive dysfunction caused by surgery. The effect of rapamycin administration on learning and memory function was determined using a CFC assessment, which was also used to measure hippocampal-dependent learning and memory. It was found that surgical injury significantly reduced the percentage freezing time $(\mathrm{P}<0.05 ; \mathrm{n}=8)$, however, rapamycin pretreatment significantly compromised the decreased freezing time caused by surgery $(60.0 \pm 8.1 \%$ in Sur+rapa group, vs. $43.4 \pm 8.0 \%$ in the Sur group), as shown in Fig. $8(\mathrm{P}<0.05 ; \mathrm{n}=8)$. Rapamycin treatment had no significant effect in the normal mice $(\mathrm{P}>0.05$; $\mathrm{n}=8$ ). These data suggested that rapamycin treatment rescued the impairment of hippocampal-dependent memory induced by surgery.

\section{Discussion}

In the present study, a mouse model was used to investigate the cellular roles of mTOR signaling in the central nervous system in response to surgical intervention. Surgical trauma was found to induce the activation of mTOR signaling with accumulation of $\mathrm{A} \beta_{1-42}$ and excessive tau protein phosphorylation in the hippocampus. Inhibition of the mTOR signaling pathway using the mTOR inhibitor, rapamycin, effectively reduced the levels of $A \beta_{1-42}$ and tau protein phosphorylation triggered by surgery, and attenuated hippocampal-dependent memory impairment. Therefore, the present study demonstrated that surgical stimuli may lead to excessive activity of 
A Day1 Day3 Day7
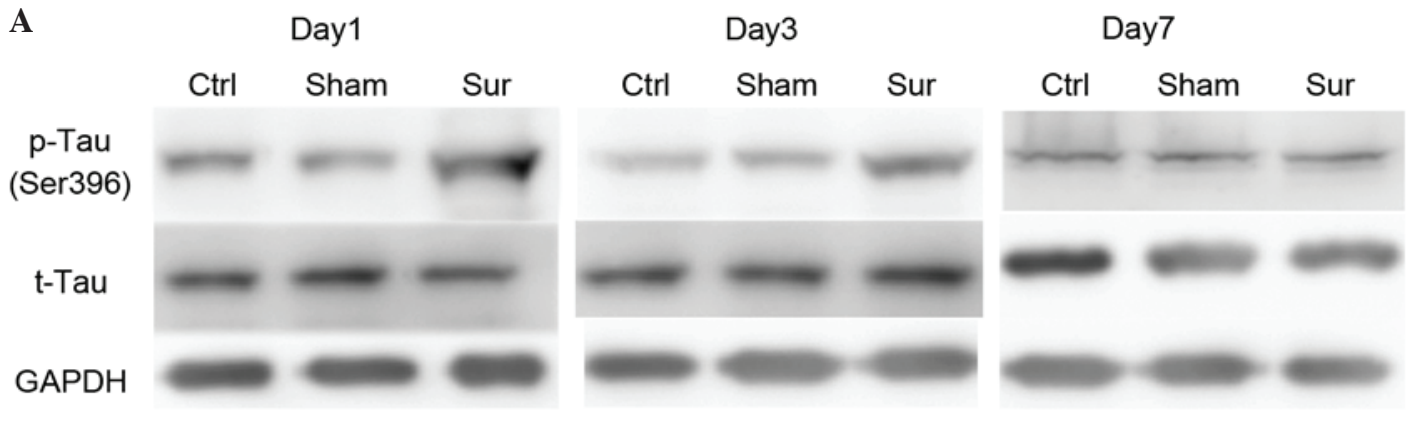

B

C
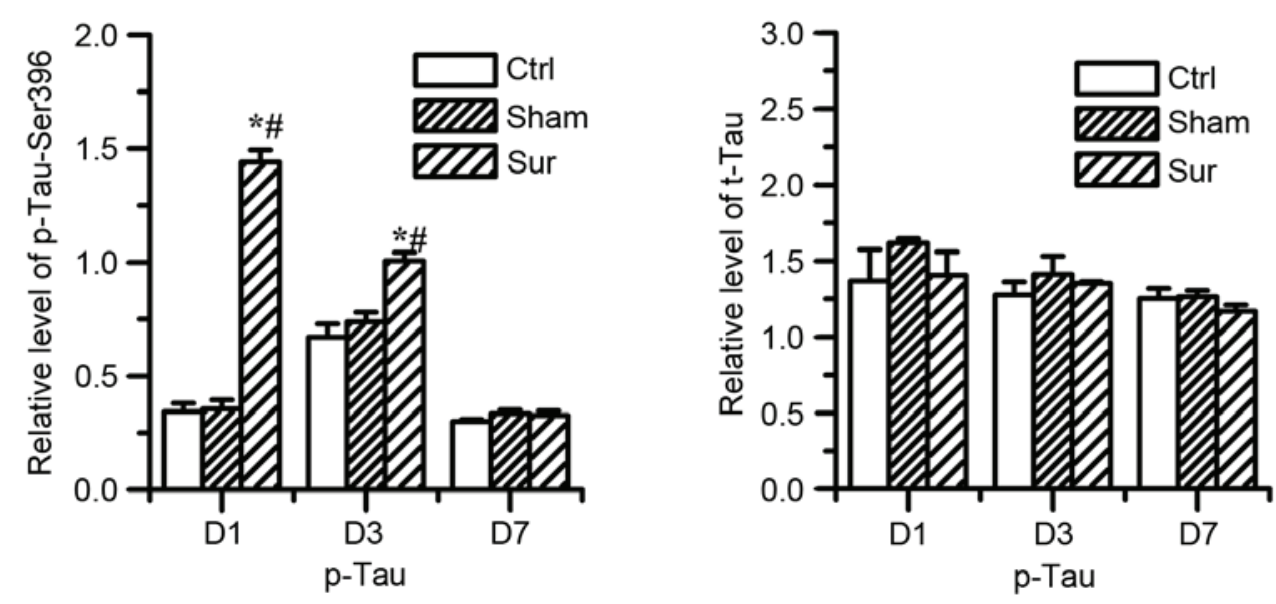

Figure 4. Effects of orthopedic surgery on the protein levels of tau in the hippocampus. The expression levels of p-tau and tau was determined using western blot analysis on days 1, 3 and 7 post-surgery. (A) Representative western blot results show the changes in the protein levels of p-tau (Ser396) and t-tau. (B) Quantitative analysis indicated that the level of p-tau (Ser396) significantly increased on days 1 and 3 post-surgery ( ${ }^{*} \mathrm{P}<0.05$, vs. Ctrl; ${ }^{\#} \mathrm{P}<0.05$, vs. Sham). (C) Levels of t-tau remained unchanged in all groups In the Sham group, no significant difference was found in the level of p-tau (Ser396) following isoflurane anesthesia, compared with the Ctrl group. (P>0.05 vs. Ctrl). Values are presented as the mean \pm standard deviation ( $\mathrm{n}=8$ for each group). Ctrl, control group; Sham, sham surgery group; Sur, surgery group; p-, phosphorylated; D1, day 1; D3, day 3; D7, day 7.

mTOR, and hyperactivity of mTOR promoted the accumulation of $A \beta_{1-42}$ and phosphorylation of tau protein, which are involved in memory and cognitive impairment.

In the present study, CFC assessments were used to evaluate cognitive function in the animals. Several studies in animals have shown that the hippocampus is critical in $\operatorname{CFC}(23,24)$, and CFC assessment has become a common method for investigating hippocampal-dependent associative memory in models of POCD $(18,25)$. In previous studies, surgical trauma has been found to be associate with impaired cognitive function $(20,26)$. In the present study, it was found that orthopedic surgery induced similar effects. Compared with the control group and sham surgery group, mice in the surgery group had a lower percentage of freezing time on day 1 and day 3 following surgery, which indicated that surgery led to hippocampal-dependent memory impairment. This was consistent with previous studies in which surgical trauma was associated with reduced freezing time $(25,27)$.

Although orthopedic surgery has been found to be associated with cognitive dysfunction, the effect of anesthetics in POCD remain controversial. In certain studies, volatile anesthetics have been shown to impair learning and memory ability. In addition, no differences have been found in the occurrence of POCD between surgery with and without general anesthesia (3). The present study found that isoflurane led to no marked reduction in the percentage of freezing time, compared with the control group, and this may be associated with the duration of isoflurane exposure.

Rapamycin, an mTOR allosteric inhibitor, has been shown to prevent the cognitive deficits induced by pathological damage. Majumder et al (28) found that rapamycin can ameliorate age-dependent cognitive deficits by reducing interleukin- $1 \beta$ (IL-1 $\beta$ ) and enhancing $\mathrm{N}$ methyl $\mathrm{D}$ aspartate signaling. In addition, rapamycin administration can attenuate cognitive deficits by ameliorating $\beta$-amyloid and tau pathology $(14,29,30)$. In the present study, it was found that rapamycin significantly alleviated the hippocampal-dependent memory impairment induced by surgical trauma. This suggested that surgery may induce the hyperactivity of mTOR signaling, which may be involved in the postoperative cognitive deficits.

mTOR is a highly conserved serine/threonine kinase. It is critical in controlling metabolism, survival, protein synthesis and phosphorylation via its downstream targets, including p70S6K and 4E-BP1. Previous studies have suggested that the role of mTOR signaling in protein homeostasis (synthesis and autophagic degradation) appears to be particularly important in the brain in learning and memory function. Synaptic plasticity is considered important in learning and memory, and the activation of mTOR can act directly at synapses to promote the synthesis of proteins, which is necessary to facilitate 
A

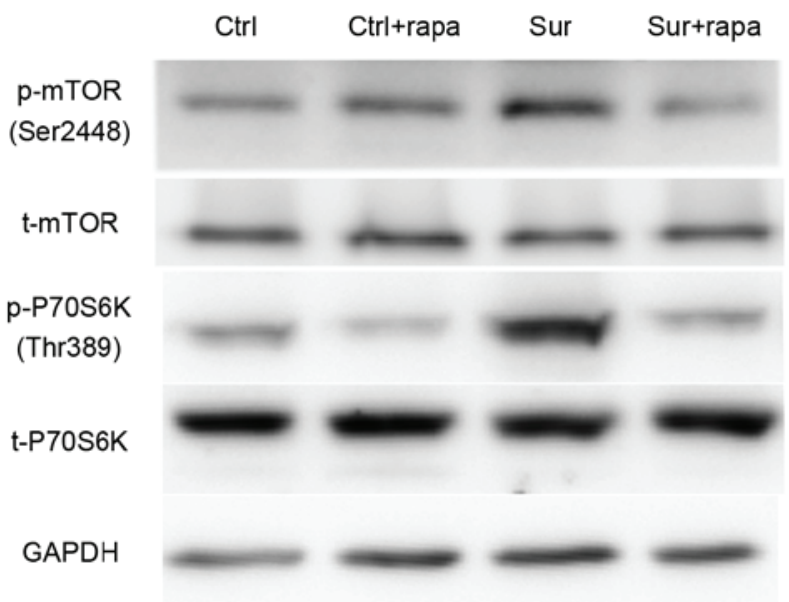

B

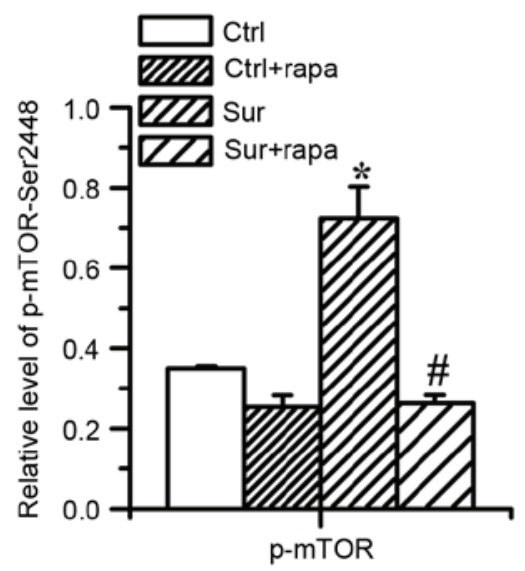

D

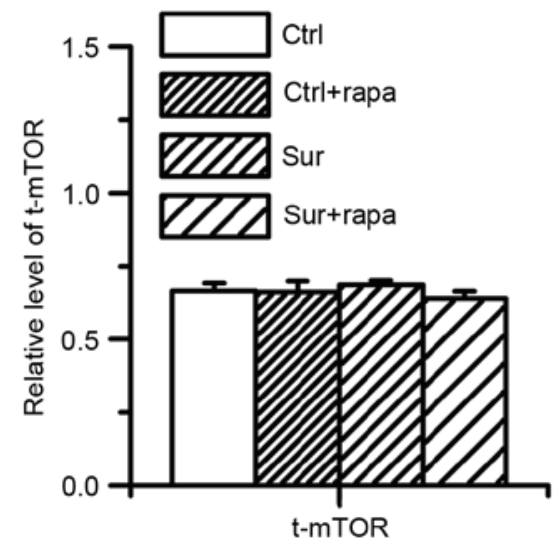

C

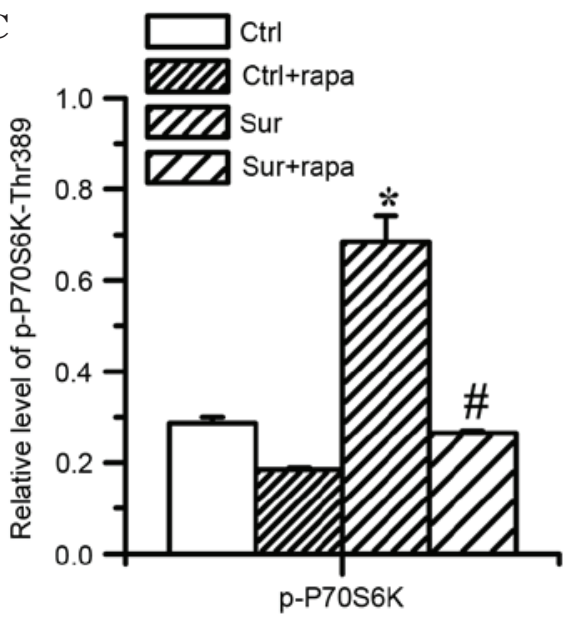

$\mathbf{E}$

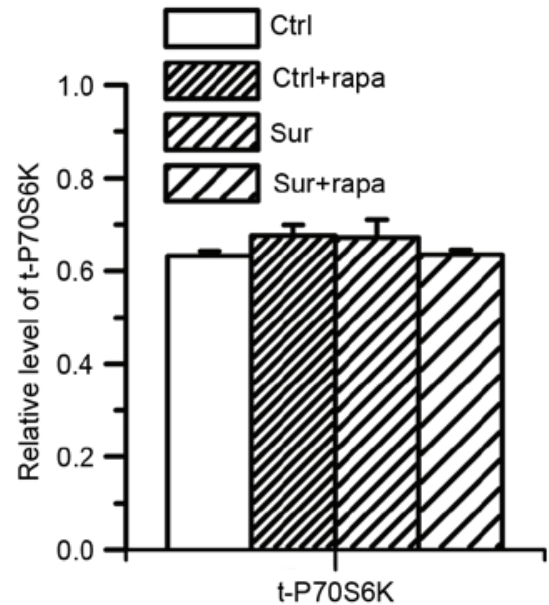

Figure 5. Effects of rapamycin pretreatment on activation of mTOR signaling in the hippocampus following surgery. The expression levels of mTOR and p70S6K were determined using western blot analysis on day 1 post-surgery. (A) Typical western blot results show changes in the expression levels of p-mTOR (Ser2448), t-mTOR, p-p70S6K (Thr396) and t-p70S6K. (B and C) Image analysis of band densities indicated that surgery significantly increased the expression levels of p-mTOR (Ser2448) and p-p70S6K (Thr396), ("P<0.05, vs. Ctrl). (B and C) Pretreatment with rapamycin significantly reduced the surgery-induced phosphorylation of p-mTOR (Ser2448) and p-p70S6K (Thr396) on day 1 post-surgery, $\left({ }^{*} \mathrm{P}<0.05\right.$, vs. Surg). (D and E) Pretreatment with rapamycin did not alter the protein expression levels of $\mathrm{t}-\mathrm{mTOR}$ or $\mathrm{t}$-p70S6K. Values are presented as the mean \pm standard deviation ( $\mathrm{n}=8$ for each group). Ctrl, control group; Ctrl+rapa, normal group with rapamycin treatment. Surg, surgery group; Sur+rapa, surgery group with rapamycin pretreatment; t-, total; p-, phosphorylated. 


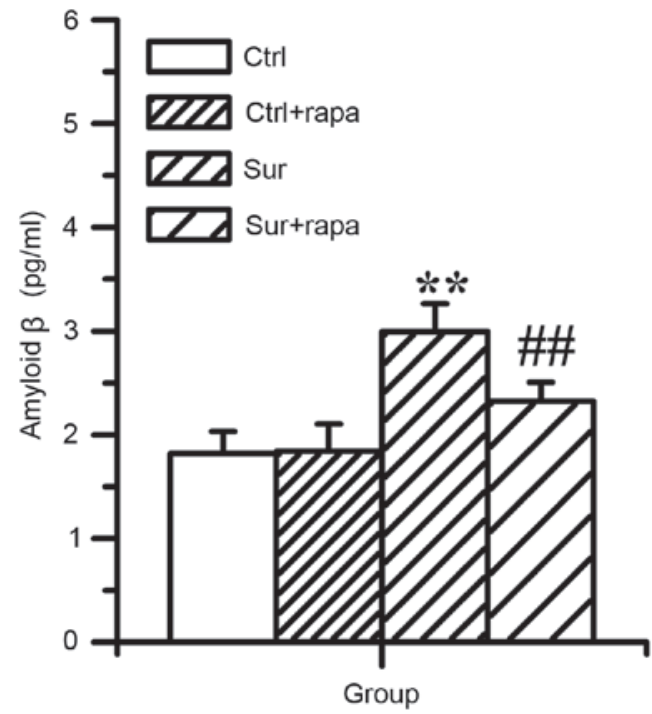

Figure 6. Effects of pretreatment of rapamycin on the levels of $A \beta_{1-42}$ in the hippocampus following surgery. The expression of $A \beta_{1-42}$ was determined using an enzyme-linked immunosorbent assay 1 day following surgery. Compared with the Ctrl group, the level of $A \beta_{1-42}$ was significantly elevated $\left({ }^{* *} \mathrm{P}<0.01\right.$, vs. $\left.\mathrm{Ctrl}\right)$. In the Sur+rapa group, the level of $\mathrm{A} \beta_{1-42}$ significantly reduced on day 1 post-surgery, compared with the Sur group $\left({ }^{\# \#} \mathrm{P}<0.01\right.$ vs. Sur) However, rapamycin treatment did not affect level of $A \beta_{1-42}$ in the Ctrl mice ( $P>0.05$, vs. Ctrl). Values are presented as the mean \pm standard deviation $\left(\mathrm{n}=8\right.$ for each group). $\mathrm{A} \beta_{1-42}$, amyloid $\beta ; \mathrm{Ctrl}$, control group; $\mathrm{Ctrl}+\mathrm{rapa}$, normal group with rapamycin treatment. Sur, surgery group; Sur+rapa, surgery group with rapamycin pretreatment.

plasticity. Hoeffer et al (31) found that the enhanced activity of mTORC1 in mice with FK-506 binding protein 12 removed from hippocampal neurons, improved memory on assessment of hippocampus-dependent memory. The inhibition of mTORC1 has also been shown to disrupt the consolidation of memories, including hippocampus-dependent spatial memory and auditory cortex-dependent memory $(32,33)$. By contrast, increasing mTORC1 activity can also disrupt memory processing. Increasing hippocampal mTORC1 activity by increasing excitatory neurotransmission in the hippocampus disrupts the formation of hippocampus-dependent memory (34). Previous observations have suggested that doses of rapamycin shown to attenuate pathology in disease models may be below the threshold to disrupt memory formation and may benefit health without detrimental cognitive effects $(13,14,35)$. Caccamo et al (13) suggested that there may be an optimal window of mTOR signaling for learning and memory. The present study is the first, to the best of our knowledge, to show that the levels of phospho-mTOR at Ser 2448 and phospho-p70S6K at Thr396 were increased in the hippocampi of mice following orthopedic surgery, suggesting that the mTOR/p70S6K signaling pathway was activated by surgical intervention.

As a well known mTOR inhibitor, the present study also determined the effect of rapamycin pretreatment on activation of the mTOR/p70S6K pathway. The levels of phospho-mTOR at Ser 2448 and phosphor-p70S6K at Thr396 were significantly reduced in the hippocampus of the rapamycin-treated mice, compared with the untreated mice following surgery. However, no significant difference in the levels of total mTOR or p70S6K were observed. The results of the present study
A

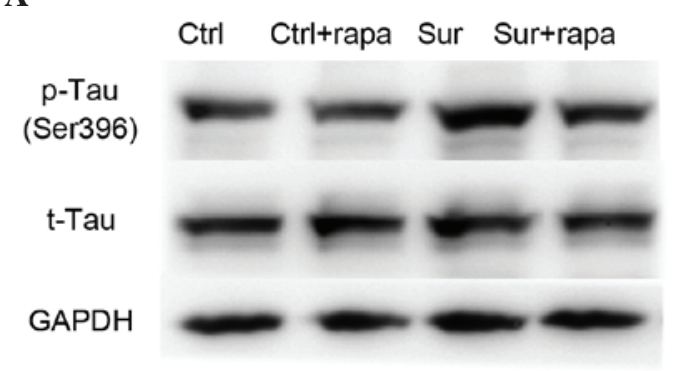

B

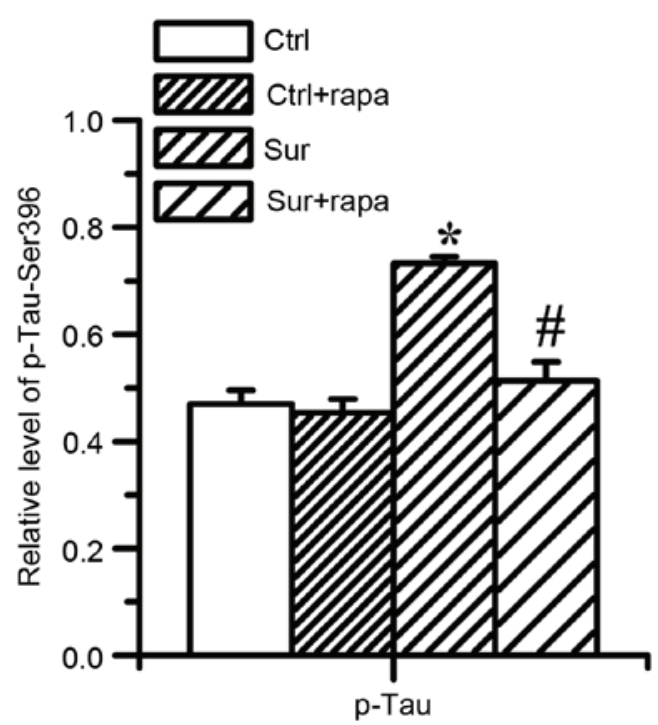

C

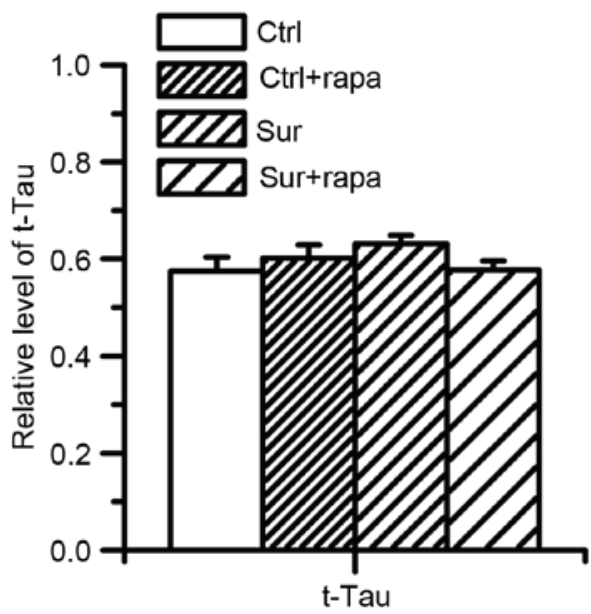

Figure 7. Effects of pretreatment with rapamycin on the levels of tau protein in the hippocampus following surgery. The expression of p-tau and tau protein was determined by western blot analysis 1 day following surgery. (A) Representative immunoblots show changes in the protein levels of p-tau (Ser396) and t-tau. (B) Quantitative analysis indicated that surgery significantly increased the level of p-tau (Ser396; 'P<0.05, vs. Ctrl). In the Sur+rapa group, the levels of p-tau (Ser396) were significantly reduced 1 day following surgery, compared with the surgery group $\left({ }^{\sharp} \mathrm{P}<0.05\right.$, vs. Sur $)$, but did not alter the expression of t-tau ( $P>0.05$, vs. Sur). (C) Rapamycin treatment did not affect the levels of $t$-tau or p-tau in the control mice ( $P>0.05$, vs. Ctrl). Values are presented as the mean \pm standard deviation ( $\mathrm{n}=8$ for each group). $\mathrm{A} \beta_{1-42}$, amyloid $\beta$; Ctrl, control group; Ctrl+rapa, normal group with rapamycin treatment. Sur, surgery group; Sur+rapa, surgery group with rapamycin pretreatment; t-, total; p-, phosphorylated. 


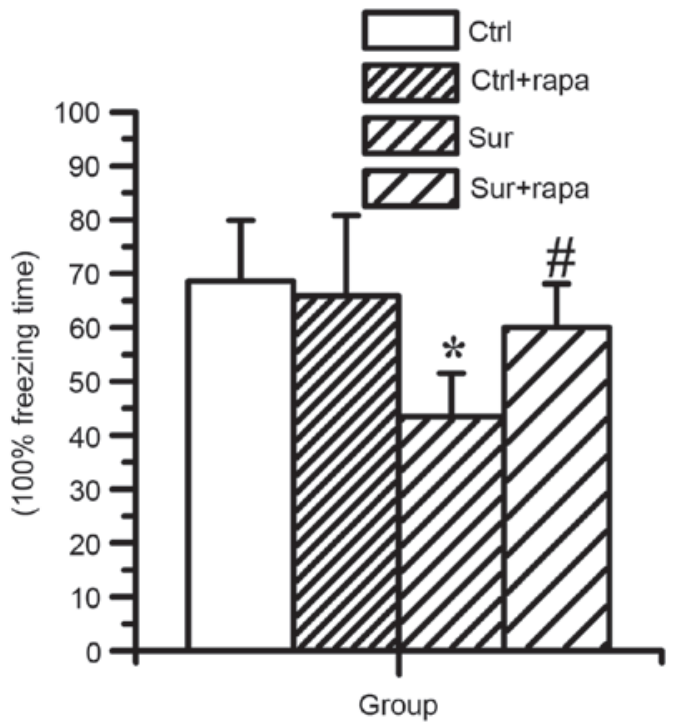

Figure 8. Effects of pretreatment with rapamycin on hippocampal-dependent memory. Surgical injury significantly reduced the percentage of freezing time $(\mathrm{P}<0.05$, vs. Ctrl). Pretreatment rapamycin significantly increased the freezing time of the Sur+rap group $(59.47 \pm 14.38 \%)$, compared with the Sur group ( $43.47 \pm 8.02$ of Sur group; $\mathrm{P}<0.05)$. No difference in freezing time was found in the normal mice with rapamycin treatment, compared with the $\mathrm{Ctr}$ group $(\mathrm{P}>0.05)$. Values are presented as the mean \pm standard deviation $(\mathrm{n}=8$ for each group). ( $\mathrm{P}<0.05$, vs. $\mathrm{Ctrl} ;{ }^{*} \mathrm{P}<0.05$, vs. Sur). Ctrl, normal contro group; Ctrl+rapa, normal group with rapamycin treatment. Sur, surgery group; Sur+rapa, surgery group with rapamycin pretreatment.

demonstrated that the increase in mTOR/p70S6K signaling in the hippocampus occurred following a traumatic stimulus, and the enhancement of mTOR/p70S6K signaling may be a key contributor to the postoperative cognitive deficits.

For decades, the hallmarks of AD pathophysiology have primarily been intracellular neurofibrillary tangles and extracellular senile amyloid plaques (36). The intraneuronal accumulation of soluble $A \beta_{1-42}$ has been shown to be a good predictor of AD pathogenesis (37). Microtubule-associated tau, as the primary constituent of neurofibrillary tangles, binds to and stabilizes microtubules in neurons. A previous study found correlations between cognitive decline and pathological markers of $\mathrm{AD}$, including the production of $\mathrm{A} \beta_{1-42}$ and the hyperphosphorylation of tau, in the hippocampus of mice following major surgery (38). Another previous study demonstrated that POCD correlated with increased levels of the AD biomarker, $A \beta_{1-42}$, in patients following cardiac surgery with cardiopulmonary bypass (39). Previous reports have shown that $\mathrm{mTOR} / \mathrm{p} 70 \mathrm{~S} 6 \mathrm{~K}$ signaling regulates the synthesis of $\beta$-amyloid and the translation of tau protein $(13,14,29,40)$. In addition to regulating tau translation, previous studies have shown that the mTOR/p70S6K pathway also modulates tau phosphorylation, directly and indirectly $(29,41)$. Therefore, the present study hypothesized that the activation of mTOR may involve $\beta$-amyloid accumulation and tau phosphorylation following surgery. Based on this, the present study analyzed the expression of $A \beta_{1-42}$ and the level of tau phosphorylation in the hippocampus. Increases in the two were found in this region on day 1 and day 3 following surgery, accompanied by the activation of mTOR. In addition, in order to determine whether the hyperactivation of mTOR induced $A \beta_{1-42}$ accumulation and tau phosphorylation, the effect of rapamycin on levels of $A \beta_{1-42}$ and tau phosphorylation were examined in the hippocampus of mice following surgery. It was found that rapamycin treatment attenuated $\mathrm{A} \beta_{1-42}$ accumulation and decreased the levels of phospho-tau at Thr396, however, no changes in total tau were observed. These results suggested that the mTOR-mediated synthesis of $A \beta_{1-42}$ and phosphorylation of tau was involved in POCD.

Although the present study demonstrated that surgical injury upregulated the activity of mTOR signaling, and then induced $\beta$-amyloid accumulation and tau phosphorylation, there remain unanswered questions. It is difficult to determine how trauma induces the hyperactivity of mTOR signaling. There is evidence that mTORC1 can be regulated by amino acids, growth factors, inflammatory mediators, hypoxia and DNA damage. The neuroinflammation induced by trauma may be involved in the hyperactivity of the mTOR signal. Previous investigations in different tissues have shown that surgery induces inflammatory responses, which are key in leading to cognitive change and decline (42). For example, surgery increases the levels of cytokines in neural tissue, including tumor necrosis factor- $\alpha$ (TNF- $\alpha$ ) and IL-1 $\beta$, the increases of which are associated with cognitive decline $(25,43)$. These proinflammatory cytokines, as with TNF- $\alpha$, have been indicated as upregulators in mTORC. Proinflammatory cytokines induce the inhibitor of $\kappa \mathrm{B}$ kinase $\beta$ (IKK $\beta$ ), which phosphorylates tuberous sclerosis 1 (TSC1) leading to TSC1/2 inhibition. When TSC1/2 is inhibited, it increases the activity of mTORC1 (44). The present study did not investigate the autophagy mediated by mTOR, and future investigations are required to determine whether trauma activates mTOR through the IKK $\beta / \mathrm{TSC} 1 / 2$ signal, the effect of surgical trauma on autophagy and the effect of mTOR in this process.

In conclusion, the present study found that orthopedic surgery may activate mTOR signaling within the hippocampus, and the hyperactivity of mTOR promoted the accumulation of $\mathrm{A} \beta_{1-42}$ and excessive phosphorylation of the tau protein. Inhibiting the hyperactivity of mTOR may reduce the production of these misfolded protein, and alleviate memory and cognitive impairment. These findings provide novel insight into the signaling transduction mechanisms in the development of postoperative cognitive dysfunction and may represent a potential therapeutic target to treat or prevent the development of POCD.

\section{Acknowledgements}

This study was supported by the National Natural Science Foundation of China (grant. no. 81171025/H0902).

\section{References}

1. Moller JT, Cluitmans P, Rasmussen LS, Houx P, Rasmussen H, Canet J, Rabbitt P, Jolles J, Larsen K, Hanning CD, et al: Long-term postoperative cognitive dysfunction in the elderly ISPOCD1 study. ISPOCD investigators. International Study of Post-Operative Cognitive Dysfunction. Lancet 351: 857-861, 1998.

2. Rubens FD, Boodhwani M and Nathan H: Interpreting studies of cognitive function following cardiac surgery: A guide for surgical teams. Perfusion 22: 185-192, 2007. 
3. Newman S, Stygall J, Hirani S, Shaefi S and Maze M: Postoperative cognitive dysfunction after noncardiac surgery: A systematic review. Anesthesiology 106: 572-590, 2007.

4. Vacas S, Degos V, Feng X and Maze M: The neuroinflammatory response of postoperative cognitive decline. $\mathrm{Br}$ Med Bull 106 161-78, 2013.

5. Krenk L, Rasmussen LS and Kehlet H: New insights into the pathophysiology of postoperative cognitive dysfunction. Acta Anaesthesiol Scand 54: 951-956, 2010.

6. Wang C, Yu JT, Miao D, Wu ZC, Tan MS and Tan L: Targeting the mTOR signaling network for Alzheimer's disease therapy. Mol Neurobiol 49: 120-135, 2014.

7. Hoeffer CA and Klann E: mTOR signaling: At the crossroads of plasticity, memory and disease. Trends Neurosci 33: 67-75, 2010

8. Banko JL, Merhav M, Stern E, Sonenberg N, Rosenblum K and Klann E: Behavioral alterations in mice lacking the translation repressor 4E-BP2. Neurobiol Learn Mem 87: 248-256, 2007.

9. Ehninger D, Han S, Shilyansky C, Zhou Y, Li W, Kwiatkowski DJ, Ramesh V and Silva AJ: Reversal of learning deficits in a Tsc2+/mouse model of tuberous sclerosis. Nat Med 14: 843-848, 2008

10. Garelick MG and Kennedy BK: TOR on the brain. Exp Gerontol 46: $155-163,2011$

11. Heras-Sandoval D, Pérez-Rojas JM, Hernández-Damián J and Pedraza-Chaverri J: The role of PI3K/AKT/mTOR pathway in the modulation of autophagy and the clearance of protein aggregates in neurodegeneration. Cell Signal 26: 2694-2701, 2014.

12. Li X, Alafuzoff I, Soininen H, Winblad B and Pei JJ: Levels of mTOR and its downstream targets 4E-BP1, eEF2, and eEF2 kinase in relationships with tau in Alzheimer's disease brain. FEBS J 272 4211-4220, 2005.

13. Caccamo A, Majumder S, Richardson A, Strong R and Oddo S Molecular interplay between mammalian target of rapamycin (mTOR), amyloid-beta, and Tau: Effects on cognitive impairments. J Biol Chem 285: 13107-13120, 2010.

14. Spilman P, Podlutskaya N, Hart MJ, Debnath J, Gorostiza O, Bredesen D, Richardson A, Strong R and Galvan V: Inhibition of mTOR by rapamycin abolishes cognitive deficits and reduces amyloid-beta levels in a mouse model of Alzheimer's disease. PLoS One 5: e9979, 2010

15. Cao XZ, Ma H, Wang JK, Liu F, Wu BY, Tian AY, Wang LL and Tan WF: Postoperative cognitive deficits and neuroinflammation in the hippocampus triggered by surgical trauma are exacerbated in aged rats. Prog Neuropsychopharmacol Biol Psychiatry 34: $1426-1432,2010$

16. Laplante M and Sabatini DM: mTOR signaling. Cold Spring Harb Perspect Biol 4(2), 2012

17. Lee DF, Kuo HP, Chen CT, Hsu JM, Chou CK, Wei Y, Sun HL, Li LY, Ping B, Huang WC, et al: IKK beta suppression of TSC1 links inflammation and tumor angiogenesis via the mTOR pathway. Cell 130: 440-455, 2007.

18. Degos V, Vacas S, Han Z, van Rooijen N, Gressens P, Su H, Young WL and Maze M: Depletion of bone marrow-derived macrophages perturbs the innate immune response to surgery and reduces postoperative memory dysfunction. Anesthesiology 118: 527-536, 2013.

19. Vizcaychipi MP, Xu L, Barreto GE, Ma D, Maze M and Giffard RG: Heat shock protein 72 overexpression prevents early postoperative memory decline after orthopedic surgery under general anesthesia in mice. Anesthesiology 114: 891-900, 2011

20. Vacas S, Degos V, Tracey KJ and Maze M: High-mobility group box 1 protein initiates postoperative cognitive decline by engaging bone marrow-derived macrophages. Anesthesiology 120: 1160-1167, 2014.

21. Erlich S, Alexandrovich A, Shohami E and Pinkas-Kramarski R Rapamycin is a neuroprotective treatment for traumatic brain injury. Neurobiol Dis 26: 86-93, 2007.

22. Tang P, Hou H, Zhang L, Lan X, Mao Z, Liu D, He C, Du H and Zhang L: Autophagy reduces neuronal damage and promotes locomotor recovery via inhibition of apoptosis after spinal cord injury in rats. Mol Neurobiol 49: 276-287, 2014

23. Broadbent NJ, Squire LR and Clark RE: Spatial memory, recognition memory, and the hippocampus. Proc Natl Acad Sci USA 101: 14515-14520, 2004.

24. Maren S, Phan KL and Liberzon I: The contextual brain: Implications for fear conditioning, extinction and psychopathology. Nat Rev Neurosci 14: 417-428, 2013.

25. Cibelli M, Fidalgo AR, Terrando N, Ma D, Monaco C, Feldmann M, Takata M, Lever IJ, Nanchahal J, Fanselow MS and Maze M: Role of interleukin-1beta in postoperative cognitive dysfunction. Ann Neurol 68: 360-368, 2010
26. Terrando N, Eriksson LI, Ryu JK, Yang T, Monaco C, Feldmann M, Jonsson Fagerlund M, Charo IF, Akassoglou K and Maze M: Resolving postoperative neuroinflammation and cognitive decline. Ann Neurol 70: 986-995, 2011.

27. Hu N, Guo D, Wang H, Xie K, Wang C, Li Y, Wang C, Wang C, $\mathrm{Yu}$ Y and Wang G: Involvement of the blood-brain barrier opening in cognitive decline in aged rats following orthopedic surgery and high concentration of sevoflurane inhalation. Brain Res 1551: 13-24, 2014

28. Majumder S, Caccamo A, Medina DX, Benavides AD, Javors MA, Kraig E, Strong R, Richardson A and Oddo S: Lifelong rapamycin administration ameliorates age-dependent cognitive deficits by reducing IL-1 $\beta$ and enhancing NMDA signaling. Aging Cell 11: 326-335, 2012.

29. Caccamo A, Magri A, Medina DX, Wisely EV,López-Aranda MF, Silva AJ and Oddo S: mTOR regulates tau phosphorylation and degradation: Implications for Alzheimer's disease and other tauopathies. Aging Cell 12: 370-380, 2013.

30. Wang S, Zhou SL, Min FY, Ma JJ, Shi XJ, Bereczki E and Wu J: mTOR-mediated hyperphosphorylation of tau in the hippocampus is involved in cognitive deficits in streptozotocin-induced diabetic mice. Metab Brain Dis 29: 729-736, 2014.

31. Hoeffer CA, Tang W, Wong H, Santillan A, Patterson RJ, Martinez LA, Tejada-Simon MV, Paylor R, Hamilton SL and Klann E: Removal of FKBP12 enhances mTOR-Raptor interactions, LTP, memory, and perseverative/repetitive behavior. Neuron 60: 832-845, 2008.

32. Dash PK, Orsi SA and Moore AN: Spatial memory formation and memory-enhancing effect of glucose involves activation of the tuberous sclerosis complex-Mammalian target of rapamycin pathway. J Neurosci 26: 8048-8056, 2006.

33. Schicknick H, Schott BH, Budinger E, Smalla KH, Riedel A, Seidenbecher CI, Scheich H, Gundelfinger ED and Tischmeyer W: Dopaminergic modulation of auditory cortex-dependent memory consolidation through mTOR. Cereb Cortex 18: 2646-2658, 2008

34. Puighermanal E, Marsicano G, Busquets-Garcia A, Lutz B, Maldonado R and Ozaita A: Cannabinoid modulation of hippocampal long-term memory is mediated by mTOR signaling. Nat Neurosci 12: 1152-1158, 2009.

35. Halloran J, Hussong SA, Burbank R, Podlutskaya N, Fischer KE, Sloane LB, Austad SN, Strong R, Richardson A, Hart MJ and Galvan V: Chronic inhibition of mammalian target of rapamycin by rapamycin modulates cognitive and non-cognitive components of behavior throughout lifespan in mice. Neuroscience 223: 102-113, 2012.

36. Braak $\mathrm{H}$ and Braak E: Neuropathological stageing of Alzheimer-related changes. Acta Neuropathol 82: 239-259, 1991.

37. Blasko I, Kemmler G, Jungwirth S, Wichart I, Krampla W, Weissgram S, Jellinger K, Tragl KH and Fischer P: Plasma amyloid beta-42 independently predicts both late-onset depression and Alzheimer disease. Am J Geriatr Psychiatry 18: 973-982, 2010

38. Wan Y, Xu J, Meng F, Bao Y, Ge Y, Lobo N, Vizcaychipi MP, Zhang D, Gentleman SM, Maze M and Ma D: Cognitive decline following major surgery is associated with gliosis, $\beta$-amyloid accumulation and tau phosphorylation in old mice. Crit Care Med 38: 2190-2198, 2010.

39. Reinsfelt B, Westerlind A, Blennow K, Zetterberg H and Ricksten SE: Open-heart surgery increases cerebrospinal fluid levels of Alzheimer-associated amyloid $\beta$. Acta Anaesthesiol Scand 57: 82-88, 2013

40. Ma YQ, Wu DK and Liu JK: mTOR and tau phosphorylated proteins in the hippocampal tissue of rats with type 2 diabetes and Alzheimer's disease. Mol Med Rep 7:623-627, 2013.

41. Tang Z, Bereczki E, Zhang H, Wang S, Li C, Ji X, Branca RM, Lehtiö J, Guan Z, Filipcik P, et al: Mammalian target of rapamycin (mTor) mediates tau protein dyshomeostasis: Implication for Alzheimer disease. J Biol Chem 288: 15556-15570, 2013.

42. Lyman M, Lloyd DG, Ji X, Vizcaychipi MP and Ma D: Neuroinflammation: The role and consequences. Neurosci Res 79: $1-12,2014$

43. Terrando N, Monaco C, Ma D, Foxwell BM, Feldmann M and Maze M: Tumor necrosis factor-alpha triggers a cytokine cascade yielding postoperative cognitive decline. Proc Nat Acad Sci USA 107: 20518-20522, 2010.

44. Zhu XC, Yu JT, Jiang T and Tan L: Autophagy modulation for Alzheimer's disease therapy. Mol Neurobiol 48: 702-714, 2013. 